\section{Mechanically Assisted Exfoliation and Functionalization of Thermally Converted Graphene Sheets}

Zhong Jin, Jay R. Lomeda, B. Katherine Price, Wei Lu, $\mathrm{Yu} \mathrm{Zhu}$, and James M. Tour*

Departments of Chemistry and Mechanical Engineering and Materials Science, The Smalley Institute for Nanoscale Science and Technology, Rice University, MS-222, 6100 Main Street, Houston, Texas 77005

Received June 10, 2009

Graphene, a single-atom-thick graphitic layer, is a twodimensional carbon material that has promising applications in electronic devices ${ }^{1}$ and composite materials. ${ }^{2}$ Although compromised from the electronics perspective, the oxidized version of graphene, graphene oxide (GO), has complementary applications in composites and thin films because of its higher processability than the parent graphene. ${ }^{3}$ The successful nanotechnological use of graphene and GO depends on the separation of bulk graphitic materials into individual sheets. There have been several chemical routes developed that can produce individual exfoliated graphene sheets but they are often not scalable or they use environmentally harmful solvents. ${ }^{4,5}$ Moreover, the functionalization of graphene and GO sheets is important for modifying their properties. ${ }^{6,7}$ Here we report an efficient, environmentally friendly and potentially scalable one-step method to peel individual sheets from thermally converted graphene (TCG, $<5 \%$ oxygen content, obtained by the thermal expansion of $\mathrm{GO}$ ) and concomitantly functionalize the predominantly graphene sheets with various aryl-addends. Our exfoliation and functionalization process involves grinding TCG in an agate mortar and pestle with an aryl diazonium salt and potassium carbonate (Scheme 1).

The solvent used was 1-octyl-3-methylimidazolium tetrafluoroborate $\left(\mathrm{OMIBF}_{4}\right)$, an ionic liquid (IL). ILs

*Corresponding author.

(1) Novoselov, K. S.; Geim, A. K.; Morozov, S. V.; Jiang, D.; Zhang, Y.; Dubonos, S. V.; Grigorieva, I. V.; Firsov, A. A. Science 2004, 306, 666.

(2) Stankovich, S.; Dikin, D. A.; Dommett, G. H. B.; Kohlhaas, K. M.; Zimney, E. J.; Stach, E. A.; Piner, R. D.; Nguyen, S. T.; Ruoff, R. S. Nature 2006, 442, 282

(3) (a) Eda, G.; Fanchini, G.; Chhowalla, M. Nat. Nanotechnol. 2008, 3, 270. (b) Lotya, M.; Hernandez, Y.; King, P. J.; Smith, R. J.; Nicolosi, V.; Karlsson, L. S.; Blighe, F. M.; De, S.; Wang, Z.; McGovern, I. T.; Duesberg, G. S.; Coleman, J. N. J. Am. Chem. Soc. 2009, 131, 3611.

(4) Li, X. L.; Wang, X. R.; Zhang, L.; Lee, S. W.; Dai, H. J. Science 2008, 319, 1229

(5) Hernandez, Y.; et al. Nat. Nanotechnol. 2008, 3, 563.

(6) Jiang, D. E.; Sumpter, B. G.; Dai, S. J. Phvs. Chem. B 2006, 110, 23628.

(7) Bekyarova, E.; Itkis, M. E.; Ramesh, P.; Berger, C.; Sprinkle, M.; de Heer, W. A.; Haddon, R. C. J. Am. Chem. Soc. 2009, 131, 1336. have been studied extensively, ${ }^{8}$ and we found them to be attractive for functionalizing single-walled carbon nanotubes (SWCNTs) because of the their ability to exfoliate SWCNT bundles. ${ }^{9,10}$ ILs also have high boiling points $\left(>200{ }^{\circ} \mathrm{C}\right)$, virtually no vapor pressure, and can be recycled. In this report, we utilize these properties to exfoliate graphene sheets and functionalize them in situ, using diazonium chemistry. ${ }^{11,12}$ Similar to the SWCNT debundling that occurred with ILs, ${ }^{9,10}$ in the present case, the IL was particularly effective in overcoming the inherent cohesive forces between graphene sheets through the mild grinding action, thereby permitting reactions to take place throughout the sample.

The starting TCG with $<10$-layered-graphene stacks can be obtained using a well-known procedure of rapidly heating GO (synthesized by Staudenmaier method) to above $1000^{\circ} \mathrm{C}$ in a $\mathrm{H}_{2} / \mathrm{Ar}$ gas atmosphere to remove most of the oxygen content; ${ }^{13,14}$ we modified this procedure by employing $10 \% \mathrm{H}_{2}$ in $\mathrm{Ar}$ to further decrease the oxygen content from $\sim 9$ to $\sim 3 \%$ and make the structure of graphene planes more ordered. Typically, TCG $(12.0 \mathrm{mg})$ was ground together with a diazonium salt $(1.2 \mathrm{mmol})$ and $\mathrm{OMIBF}_{4}(1.0 \mathrm{~mL})$ for $10 \mathrm{~min}$. The base, $\mathrm{K}_{2} \mathrm{CO}_{3}(10.0 \mathrm{mg})$, was then added to facilitate the functionalization, ${ }^{9,10}$ and the mixture was ground for an additional $45 \mathrm{~min}$. After acetone washing, the resulting suspension was filtered on a Teflon membrane $(0.45 \mu \mathrm{m}$ pore size). To completely remove residual IL, base, and organic compounds, we subsequently redispersed the filter cake and washed it in water, alcohol, and acetone until the filtrate was clearly transparent. The product was then stored in a vacuum oven at $80{ }^{\circ} \mathrm{C}$ before characterization. The functionalized materials had a small weight gain presumably because of the functional groups grafted on the graphene sheets. The IL, $\mathrm{OMIBF}_{4}$, was chosen because of the length of the alkyl chain. In past reports, an increased alkyl chain length was shown to enhance the debundling and functionalization of SWCNTs. ${ }^{10}$ A second IL, hexylpyridinium tetrafluoroborate was shown to produce results comparable to the $\mathrm{OMIBF}_{4}$. Also, the aryl diazonium borofluoride salt can be replaced by its corresponding aniline together with sodium nitrite or isoamyl nitrite to form the diazonium salt in situ;

(8) Greaves, T. L.; Drummond, C. J. Chem. Rev. 2008, 108, 206.

(9) Fukushima, T.; Kosaka, A.; Ishimura, Y.; Yamamoto, T.; Takigawa, T.; Ishii, N.; Aida, T. Science 2003, 300, 2072.

(10) Price, B. K.; Hudson, J. L.; Tour, J. M. J. Am. Chem. Soc. 2005 , $127,14867$.

(11) Staudenmaier, L. Ber. Dtsch. Chem. Ges. 1898, 31, 1481.

(12) Lomeda, J. R.; Doyle, C. D.; Kosynkin, D. V.; Hwang, W. F.; Tour, J. M. J. Am. Chem. Soc. 2008, 130, 16201.

(13) Schniepp, H. C.; Li, J. L.; McAllister, M. J.; Sai, H.; HerreraAlonso, M.; Adamson, D. H.; Prud'homme, R. K.; Car, R.; Saville, D. A.; Aksay, I. A. J. Phvs. Chem. B 2006, 110, 8535 .

(14) McAllister, M. J.; Li, J. L.; Adamson, D. H.; Schniepp, H. C.; Abdala, A. A.; Liu, J.; Herrera-Alonso, M.; Milius, D. L.; CarO, R.; Prud'homme, R. K.; Aksay, I. A. Chem. Mater. 2007, 19, 4396. 
Scheme 1. Exfoliation and Functionalization of Graphene Sheets by Grinding TCG in ionic Liquid Using a Mortar and Pestle; Compounds $2 \mathrm{f}$ and $3 \mathrm{f}$ are Meta-Substituted

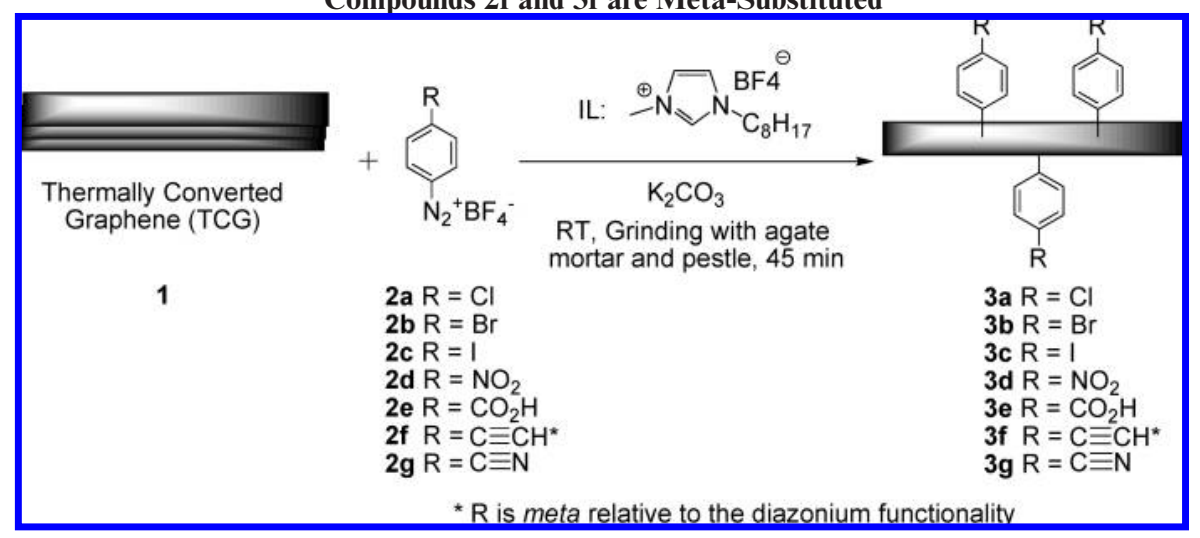

however, the efficiency of functionalization decreased slightly in these cases.

By employing different aryl diazonium salts $(\mathbf{2 a}-\mathbf{2 g})$, the addends of the functionalized materials can be conveniently customized. The degree of functionalization was monitored with X-ray photoelectron spectroscopy (XPS), thermogravimetric analysis (TGA) and Fourier transform infrared spectroscopy (FTIR). XPS analysis of products $\mathbf{3 a}-\mathbf{3 g}$ (see the Supporting Information, Figure S1), showed specific elements from the grafted aryl groups; no boron or fluorine peaks were detected, thus indicating that $\mathrm{OMIBF}_{4}$ had been completely removed from the functionalized graphene. After reaction with halide-substituted diazonium salts, the halogen peaks were detected with rarely any nitrogen peak, providing evidence that the graphene sheets had been functionalized. High-resolution XPS of $\mathbf{3 a}, \mathbf{3} \mathbf{b}$, and $\mathbf{3} \mathbf{c}$ show the atomic percentages of halogens as $\mathbf{3 a}, 2.7 \% \mathrm{Cl} ; \mathbf{3} \mathbf{b}$, $2.3 \% \mathrm{Br}$; and $3 \mathbf{c}, 1.7 \%$ I. Meanwhile, obvious nitrogen peaks can be found in $\mathbf{3 d}, 5.3 \% \mathrm{~N}$ and $\mathbf{3 g}, 3.7 \% \mathrm{~N}$.

FTIR spectra (see the Supporting Information, Figure S2) give characteristic absorbances for functional groups grafted on graphene sheets. The FTIR spectrum of 3d shows the asymmetric stretch at $1515 \mathrm{~cm}^{-1}$ and the symmetric stretch at $1342 \mathrm{~cm}^{-1}$ of the $-\mathrm{NO}_{2}$ group and $\mathrm{C}-\mathrm{N}$ stretch at $852 \mathrm{~cm}^{-1}$. Other addends were also detected: 3e, $\mathrm{OH}$ stretch $3234 \mathrm{~cm}^{-1}$ and $\mathrm{C}=\mathrm{O}$ stretch $1701 \mathrm{~cm}^{-1} ; \mathbf{3 f}, \equiv \mathrm{CH}$ stretch $3284 \mathrm{~cm}^{-1}$ and $\mathrm{C} \equiv \mathrm{C}$ stretch $2110 \mathrm{~cm}^{-1} ; \mathbf{3 g}, \mathrm{C} \equiv \mathrm{N}$ stretch $2225 \mathrm{~cm}^{-1}$.

For further investigating the degree of functionalization, TGA was completed on TCG and all products. The samples were heated to $110^{\circ} \mathrm{C}$, held for $60 \mathrm{~min}$ to remove water and remaining solvent, and then cooled to room temperature. Subsequently, the samples were heated to $850^{\circ} \mathrm{C}$ at $10^{\circ} \mathrm{C} /$ min under an $\mathrm{Ar} / \mathrm{N}_{2}$ atmosphere. The TCG overall weight loss was less than $1 \%$, indicating that most of the surface groups were removed after the original thermal expansion. The observed weight loss of all products are as follows: $\mathbf{3 a}, 14 \% ; \mathbf{3 b}, 16 \% ; \mathbf{3 c}, 21 \% ; \mathbf{3 d}$, $14 \%$; 3e, 12\%; 3f, 13\%; 3g, 26\% (see the Supporting Information, Figure S3). The onset of major weight loss was generally observed at about $250-300{ }^{\circ} \mathrm{C}$ and attributed to the thermal removal of organic functional groups.

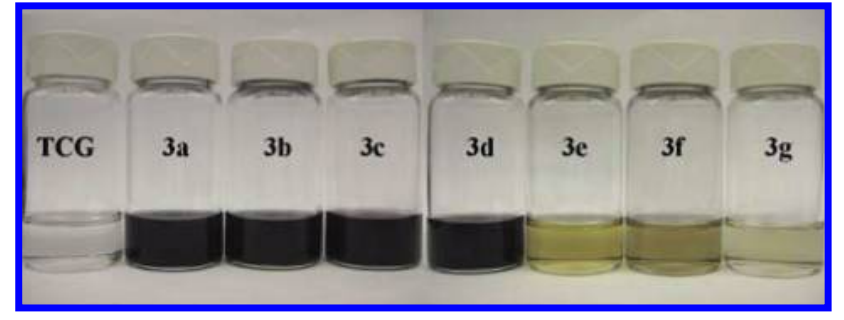

Figure 1. Photographs of supernatant solutions obtained from TCG (left vial) and functionalized graphene $(\mathbf{3 a} \mathbf{- 3}$ ) dispersed in DMF and then centrifugated at $3200 \mathrm{rpm}$ for $30 \mathrm{~min}$.

To calculate the number of functional groups, we converted the weight loss to moles (carbon) of the corresponding aryl group added and then divided by the moles of carbon remaining. The average number of aryl groups added was roughly 1 in 60 carbons; however, the weight loss of TGA is further complicated because of the possible presence of other labile groups. This degree of functionalization is moderate, leaving much of the conjugated basal graphene planes relatively intact, yet also sufficient to obtain good dispersibility of the final materials in solvent.

The solubility of graphene sheets with different functional groups in $N, N^{\prime}$-dimethylformamide (DMF) was also measured. Great increases in DMF solubility of the products were observed when compared to the TCG starting material. TCG $(10.0 \mathrm{mg})$ or functionalized graphene $(10.0 \mathrm{mg})$ were respectively dispersed in $10.0 \mathrm{~mL}$ of DMF, the suspensions were bath-sonicated (Cole-Parmer Ultrasonic Cleaner, model 08849-00, $12 \mathrm{~W}$ ) for $5 \mathrm{~min}$ and then centrifugated in an Adams Analytical centrifuge (model CT 3201) for $30 \mathrm{~min}$ at $3200 \mathrm{rpm}$. After centrifugation, the color of functionalized graphene supernatant was still dark, whereas the TCG supernatant was almost colorless and transparent. The upper halves $(5.0 \mathrm{~mL})$ of the supernatant solutions (Figure 1) were carefully taken and the solutes diluted in acetone, the solids filtered, and the filter cakes washed with acetone, vacuum-dried overnight, and weighed to calculate the solubilities of the products: TCG, $<0.01 \mathrm{mg} / \mathrm{mL} ; \mathbf{3 a}, 0.45 \mathrm{mg} / \mathrm{mL}$; 3b, $0.35 \mathrm{mg} / \mathrm{mL} ; 3 \mathbf{c}, 0.25 \mathrm{mg} / \mathrm{mL} ; 3 \mathbf{d d}, 0.30 \mathrm{mg} / \mathrm{mL} ; \mathbf{3 e}$, $0.04 \mathrm{mg} / \mathrm{mL} ; 3 \mathbf{3 f}, 0.05 \mathrm{mg} / \mathrm{mL} ; 3 \mathbf{3 g}, \sim 0.02 \mathrm{mg} / \mathrm{mL}$. Because of the influence of different functionalized addend 


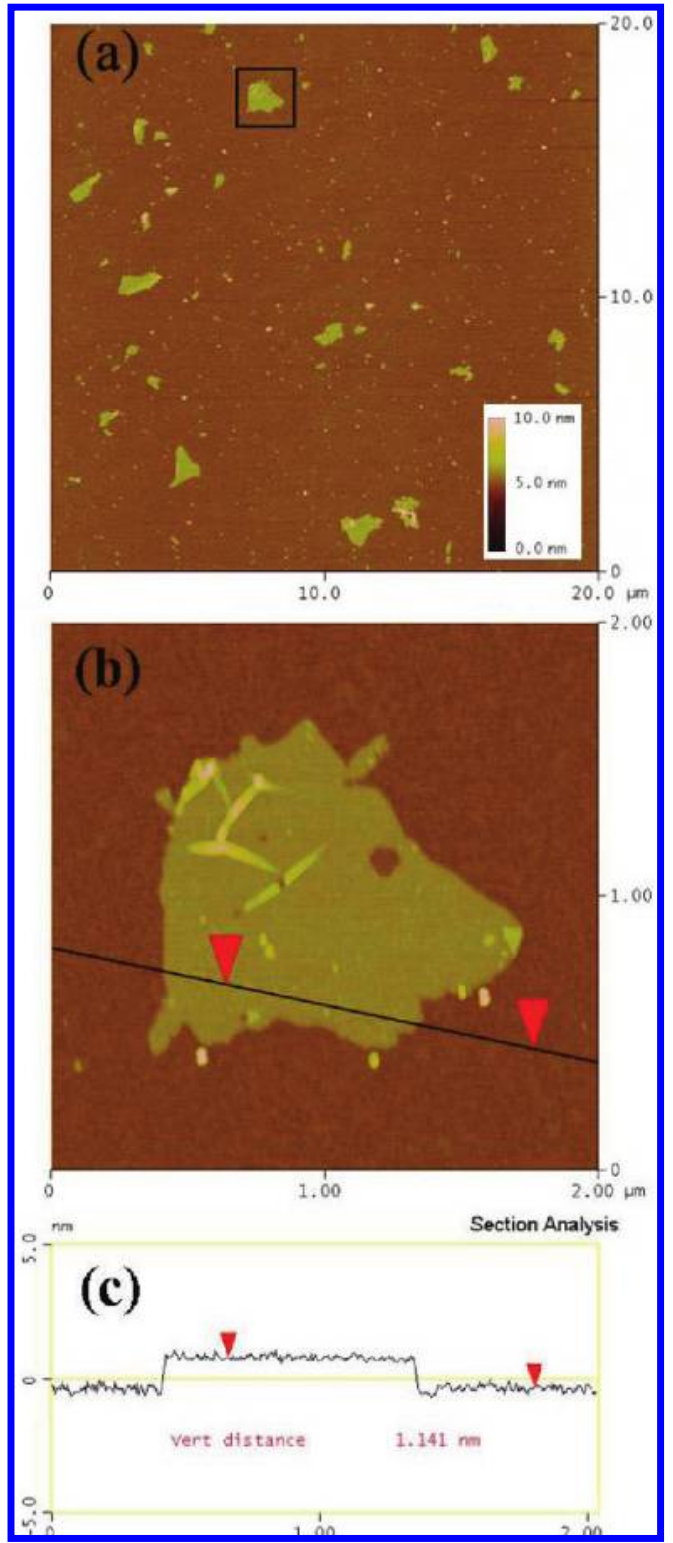

Figure 2. (a) Large-area AFM image of TCG sheets functionalized in $\mathrm{OMIBF}_{4}$ by 4-chlorobenzenediazonium tetrafluoroborate (product 3a) on a freshly cut mica surface (also see the Supporting Information, Figure S4). The measured height of graphene sheets have a range between 0.7 and $1.3 \mathrm{~nm}$ (excluding the rippled areas). Some small fragments and folds of graphene sheets can also be observed. (b) The enlargement of the boxed area from (a) with corresponding sectional analysis height data (c).

groups, the solubility of the products $\mathbf{3 e}-\mathbf{3 g}$ after the longtime centrifugation is lower than the products with halide or nitro addends $\mathbf{3 a - 3 d}$, but it is still much higher than pristine TCG. The increase and variability of the solubility in DMF showed that graphene sheets can be efficiently exfoliated and functionalized with different aryl groups in ILs by the assistance of mechanical grinding.

Tapping mode AFM was also used to characterize the functionalized graphene. Figure 2 shows a typical largearea AFM image of graphene sheets that were spincoated onto a mica surface from the supernatant solution of 3a in DMF (more AFM images can be seen in

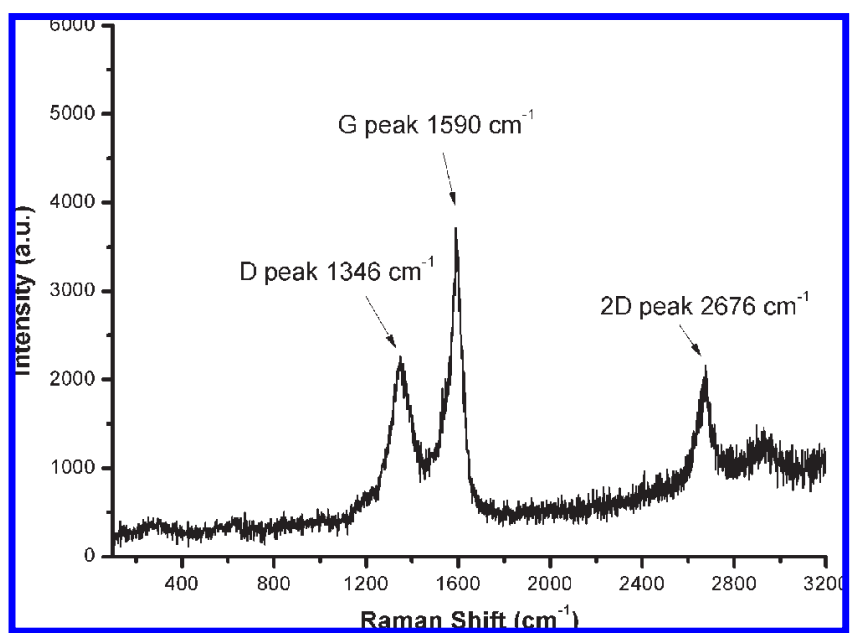

Figure 3. Raman spectrum of product 3a obtained by $514.5 \mathrm{~nm}$ excitation (Renishaw 1000).

Supporting Information S4). Ripples and folds of the graphene sheets also can be clearly seen in the AFM images. The measured height of the flat graphene sheets is between $0.7-1.3 \mathrm{~nm}$, which is in accord with the thickness of single-layer graphene sheets. ${ }^{15}$ The Raman spectrum of functionalized TCG was obtained with a $514.5 \mathrm{~nm}$ laser (Figure 3); the 2D peak shows the presence of the $\mathrm{sp}^{2}$ conjugated network in the graphene. The $D / G$ peak height ratios of functionalized graphene sheets is roughly 0.6 , which is lower than the chemical converted graphene sheets (CCG) obtained from hydrazine hydrate-reduced graphite oxide; ${ }^{16}$ this indicates the graphene plane structure of functionalized TCG was partially recovered.

In summary, single-step mechanical assisted exfoliation and functionalization of graphene using TCG and aryl-diazonium salts in ILs has been successfully demonstrated. In comparison to other methods, this process is environmentally friendly and is a potentially more scalable procedure. The graphene sheets produced here could be exploited for its unique properties that have been modified by linked aryl groups. The enhanced dispersibility in solvent could prove essential in making individual graphene sheet-based composites scaffolds.

Acknowledgment. Funding from the FAA, the US Department of Energy's Office of Energy Efficiency and Renewable Energy within the Hydrogen Sorption Center of Excellence, DE-FC-36-05GO15073, the Advanced Energy Consortium, and UT Battelle is gratefully acknowledged.

Supporting Information Available: XPS, FTIR, TGA, and AFM data of the functionalized graphene $\mathbf{3 a}-\mathbf{3 g}$ and the full citation for ref 5 (PDF). This material is available free of charge via the Internet at http://pubs.acs.org.

(15) Stankovich, S.; Piner, R. D.; Chen, X. Q.; Wu, N. Q.; Nguyen, S. T.; Ruoff, R. S. J. Mater. Chem. 2006, 16, 155.

(16) Stankovich, S.; Dikin, D. A.; Piner, R. D.; Kohlhaas, K. A.; Kleinhammes, A.; Jia, Y.; Wu, Y.; Nguyen, S. T.; Ruoff, R. S. Carbon 2007, 45, 1558. 